\title{
Sertraline improves symptoms in children and adolescents with major depressive disorder
}

Wagner KD, Ambrosini P, Rynn M, et al. Efficacy of sertraline in the treatment of children and adolescents with major depressive disorder: two randomized controlled trials. JAMA

2003;290:1033-41.

\section{What are the effects of sertraline in children and adolescents with major depressive disorder (MDD)?}

\section{METHODS}

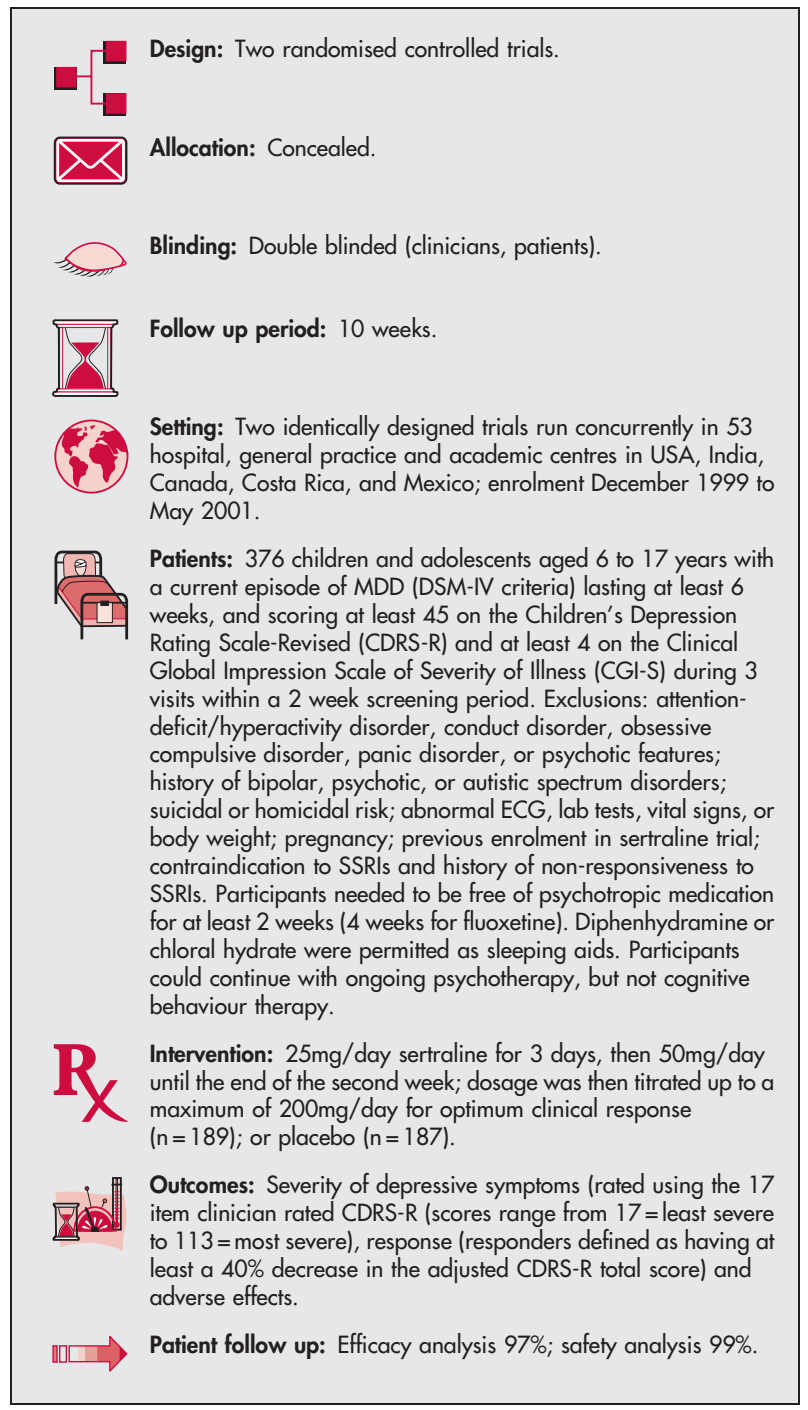

\section{MAIN RESULTS}

Sertraline significantly improved depressive symptoms compared with placebo over 10 weeks (mean change in CDRS-R score: -22.8 with sertraline $v-20.2$ with placebo; $\mathrm{p}=0.007$; analysis not by intention to treat). $69 \%$ of the sertraline group responded, compared with $59 \%$ of the placebo group $(\mathrm{p}=0.05)$. More participants in the sertraline group withdrew due to adverse effects than in the placebo

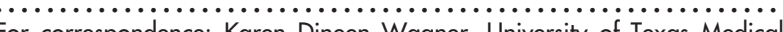
For correspondence: Karen Dineen Wagner, University of Texas Medical Branch, Department of Psychiatry and Behavioral Sciences, Division of Child and Adolescent Psychiatry, University Boulevard, Galveston, Texas, USA; kwagner@utmb.edu

Source of funding: Pfizer Inc. group (17/189 (9\%) with sertraline $v 5 / 187$ (3\%) in the placebo group; $\mathrm{p}=0.14$ (Fisher's exact, calculated from results as significance not stated in paper)). Adverse effects occurring in at least 5\% of the sertraline group, and with an incidence of at least twice that of the placebo group were: insomnia, diarrhoea, anorexia, vomiting, agitation, urinary incontinence, and purpura.

\section{CONCLUSION}

Sertraline is an effective and safe short term treatment for children and adolescents with MDD.

\section{Commentary}

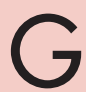

iven the high prevalence of major depressive disorder (MDD) among the young, the authors are to be commended for undertaking this research, which holds important implications for depressed children and adolescents. The current study appears particularly important given its large sample, wide age range, and use of a standard, parallel group placebo controlled design.

Responder criteria were met by $10 \%$ more of the sertraline group than the placebo group. This difference is statistically significant, and the authors suggest that it is clinically meaningful. Nevertheless, conclusions on the meaningfulness of this $10 \%$ difference should be drawn in the context of other RCTs on paediatric, as well as adult, mood, and anxiety disorders. In general, a $10 \%$ difference between an active medication and placebo is below the standard usually applied to identify treatments that are of substantial clinical benefit for mood and anxiety disorders.

The issue of whether the present findings indicate clinically meaningful benefits of sertraline for depressed children and adolescents must be evaluated in the context of several other relevant questions. Firstly, is the medication safe for youth? In light of the ongoing controversy regarding the safety of various SSRIs in paediatric populations, the present study yields reassuring findings. No evidence of treatment related differences emerged for clinically meaningful indices of adverse outcomes. Secondly, is sertraline alone just as or more efficacious than non-pharmacological or combined treatment for MDD? Based on findings that combined treatment benefits adults with chronic $M D D,{ }^{1}$ research comparing combined and single modality treatment approaches in youth is currently underway. ${ }^{2}$ Such research is all the more important given the modest difference between placebo and sertraline in the current study. Finally, the current study provides important information on acute treatment, but provides no data concerning length of treatment. Because MDD is associated with high risk for relapse or recurrence, studies are needed that compare risks and benefits of long term SSRI treatment, as opposed to placebo substitution for an initially effective SSRI. ${ }^{3}$ Such "maintenance" studies appear all the more important given the role of serotonin in both general and specific aspects of brain plasticity as it relates to stress regulation across development. ${ }^{4}$

Erin B McClure, PhD, Ellen Leibenluft, MD, Daniel S Pine, MD National Institute of Mental Health, Bethesda, Maryland, USA 1 Keller MB, McCullough JP, Klein DN, et al. A comparison of nefazodone, the cognitive behavioral-analysis system of psychotherapy, and their combination for the treatment of chronic depression. N Engl J Med 2000;342:1462-70.

2 The Treatment for Adolescents with Depression Study Team. Treatment for adolescents with depression study (TADS): rationale, design, and methods. J Am Acad Child Adolesc Psychiatry 2003;42:531-42.

3 Emslie GJ, Mayes TL. Mood disorders in children and adolescents: psychopharmacological treatment. Biol Psychiatry 2001;49:1082-90.

4 Pine DS. Treating children and adolescents with selective serotonin reuptake inhibitors: how long is appropriate? J Child Adolesc Psychopharmacol 2002;12:189-203 